\title{
Modulation of a Single Neuron Has State- Dependent Actions on Circuit Dynamics ${ }^{1,2,3}$
}

\author{
(D)Gabrielle J. Gutierrez and ${ }^{(D E v e ~ M a r d e r ~}$
}

DOI:http://dx.doi.org/10.1523/ENEURO.0009-14.2014

Volen Center for Complex Systems and Biology Department, Brandeis University, Waltham, Massachusetts 02454

\begin{abstract}
When does neuromodulation of a single neuron influence the output of the entire network? We constructed a five-cell circuit in which a neuron is at the center of the circuit and the remaining neurons form two distinct oscillatory subnetworks. All neurons were modeled as modified Morris-Lecar models with a hyperpolarizationactivated conductance $\left(\bar{g}_{h}\right)$ in addition to calcium $\left(\bar{g}_{C a}\right)$, potassium $\left(\bar{g}_{K}\right)$, and leak conductances. We determined the effects of varying $\bar{g}_{C a}, \bar{g}_{K}$, and $\bar{g}_{h}$ on the frequency, amplitude, and duty cycle of a single neuron oscillator. The frequency of the single neuron was highest when the $\bar{g}_{K}$ and $\bar{g}_{h}$ conductances were high and $\bar{g}_{C a}$ was moderate whereas, in the traditional Morris-Lecar model, the highest frequencies occur when both $\bar{g}_{K}$ and $\bar{g}_{C a}$ are high.

We randomly sampled parameter space to find 143 hub oscillators with nearly identical frequencies but with disparate maximal conductance, duty cycles, and burst amplitudes, and then embedded each of these hub neurons into networks with different sets of synaptic parameters. For one set of network parameters, circuit behavior was virtually identical regardless of the underlying conductances of the hub neuron. For a different set of network parameters, circuit behavior varied with the maximal conductances of the hub neuron. This demonstrates that neuromodulation of a single target neuron may dramatically alter the performance of an entire network when the network is in one state, but have almost no effect when the circuit is in a different state.
\end{abstract}

Key words: connectome; CPG; electrical coupling; h-conductance; half-center oscillator; Morris-Lecar model

\section{Significance Statement}

We use computational models to examine the circumstances under which modulation of a single neuron can alter the dynamics of an entire circuit. We show that under some circumstances, the circuit's behavior is robust to the neuromodulation of a single hub neuron, while under other circumstances the same neuromodulatory action of a single neuron can produce a large variety of circuit outcomes.

Received August 29, 2014; accepted October 27, 2014; First published November 12, 2014.

${ }^{1}$ Funding: National Institutes of Health Grants NS17813 and MH46742.

${ }^{2}$ The authors declare no competing financial interests.

${ }^{3}$ Author contributions: G.J.G. and E.M. designed research; G.J.G. performed research; G.J.G. and E.M. wrote the paper.

Correspondence should be addressed to Dr. Eve Marder, Volen Center for Complex Systems and Biology Department, Brandeis University, 415 South Street, Waltham, MA 02454. E-mail: marder@brandeis.edu.

G.J. Gutierrez's present address: Group for Neural Theory, Département d’Études Cognitives, École Normale Supérieure, 29 rue d’Ulm, 75005 Paris, France. DOI:http://dx.doi.org/10.1523/ENEURO.0009-14.2014

Copyright @ 2014 Gutierrez \& Marder

This is an open-access article distributed under the terms of the Creative Commons Attribution License Attribution-Noncommercial 4.0 International which permits noncommercial reuse provided that the original work is properly attributed. 


\section{Introduction}

Neuromodulators change the excitability of individual neurons and can also alter the strengths of synapses by acting on one or more of the voltage-dependent conductances of network neurons (Levitan, 1978, 1988; Marder and Bucher, 2007; Stein, 2009; Harris-Warrick, 2011; Johnson et al., 2011; Marder, 2012; Marder et al., 2014; Nadim and Bucher, 2014). In so doing, neuromodulators can modify circuit dynamics (Morgan et al., 2000; Blitz and Nusbaum, 2012; Diehl et al., 2013). In some cases, the same neuromodulator may act on many network neurons simultaneously (Harris-Warrick et al., 1998; Swensen and Marder, 2001; Harris-Warrick and Johnson, 2010; Harris-Warrick, 2011), or a neuromodulator may target one or a few neurons in a network (Nadim and Bucher, 2014).

To what extent is modulation of a single neuron in an interconnected network likely to alter the activity of many or all neurons of the network, and how might this depend on the properties of the modulated neuron and other parameters in the network? Under some conditions, changes in the intrinsic properties of a single neuron might be subordinate to the network inputs it receives, while under other conditions, changes in the intrinsic properties of a single neuron may have more of an influence on its output as well as the output produced by other network neurons.

To address these questions, we look at the effects of neuromodulation of a single hub neuron at the center of a five-cell coupled-oscillator network. This network is loosely motivated by the core connectivity within the crustacean stomatogastric ganglion, and consists of two reciprocally inhibitory half-center oscillators, with different intrinsic frequencies, connected via electrical synapses through an intrinsically oscillatory hub neuron (Gutierrez and Marder, 2013; Gutierrez et al. 2013). This five-cell circuit can show a variety of different patterns of coordination among the neurons as a function of the chemical and electrical synapses. We investigated the effects of neuromodulation of the hub neuron on the patterns of activity produced in the five-cell network. The neurons used in this study are Morris-Lecar model neurons with an added h-conductance (LoFaro et al. 1994). As a first step in understanding the role of modulation of the hub neuron, we characterize the behavior of this model neuron as a function of its maximal conductances.

This raises a series of timely questions about the circumstances under which having the connectivity diagram or "connectome" is, as a first approximation, sufficient to determine circuit dynamics.

\section{Materials and Methods}

Morris-Lecar model neuron with h-conductance

Neurons were modeled as Morris-Lecar (Morris and Lecar, 1981; LoFaro et al., 1994), single-compartment neurons with the addition of a hyperpolarization-activated conductance (h-conductance), as in Gutierrez et al. (2013). Each cell's membrane voltage, $V_{m}$, was computed by solving the equation:

$$
C_{m} \frac{d V_{m}}{d t}=-\left[I_{\text {leak }}+I_{c a}+I_{K}+I_{h}+I_{\text {elec }}+I_{\text {syn }}\right]
$$

$C_{m}$ is the membrane capacitance and is equal to $1 \mathrm{nF}$ for all neurons. Reversal potentials for the various currents are $V_{\text {leak }}=-40 \mathrm{mV}, V_{\text {ca }}=100 \mathrm{mV}, V_{\mathrm{k}}=-80 \mathrm{mV}, V_{h}=$ $-20 \mathrm{mV}, V_{\text {syn }}=-75 \mathrm{mV}$. The hyperpolarization-activated current is based on equations and parameters that were modified from Turrigiano et al. (1995). The remaining ionic currents are based on equations modified from Skinner et al. (1993). Ionic conductances $\bar{g}_{C a}$ and $\bar{g}_{K}$ were sampled from a 5 to $75 \mathrm{nS}$ range in $5 \mathrm{nS}$ intervals. The h-conductance was sampled from a 0 to $75 \mathrm{nS}$ range in 5 nS intervals. $\bar{g}_{\text {leak }}$ was set to $0.1,1$, or $10 \mathrm{nS}$.

$$
I_{\text {leak }}=\bar{g}_{\text {leak }}\left(V_{m}-V_{\text {leak }}\right)
$$

$I_{c a}=\bar{g}_{c a} M_{\infty}\left(V_{m}-V_{c a}\right), M_{\infty}=0.5\left(1+\tanh \left(V_{m}-\right.\right.$ $\left.v_{1} / v_{2}\right)$ ), where $v_{1}=0 \mathrm{mV}$ and $v_{2}=20 \mathrm{mV}$.

$I_{K}=\bar{g}_{K} N\left(V_{m}-V_{K}\right), d N / d t=\lambda_{N}\left(N_{\infty}-N\right), N_{\infty}=0.5$ $\left(1+\tanh \left(V_{m}-v_{3} / v_{4}\right)\right), \lambda_{N}=\varphi_{N} \cosh \left(V_{m}-v_{3} / 2 v_{4}\right)$, where $v_{3}=0 \mathrm{mV}$ and $v_{4}=15 \mathrm{mV}$.

$I_{h}=\bar{g}_{h} H\left(V_{m}-V_{h}\right), d H / d t=\left(H_{\infty}-H\right) / \tau_{h}, H_{\infty}=1 /$ $\left(1+\exp \left(V_{m}+v_{5} / v_{6}\right)\right), \tau_{h}=272-(-1499 /(1+\exp$ $\left.\left.\left(-V_{m}+v_{7} / v_{8}\right)\right)\right)$, where $v_{5}=78.3 \mathrm{mV}, v_{6}=10.5 \mathrm{mV}$, $v_{7}=-42.2 \mathrm{mV}, v_{8}=87.3 \mathrm{mV}$.

$M_{\infty}, N_{\infty}$, and $H_{\infty}$ are steady-state gating variables for the calcium, potassium, and hyperpolarization-activated currents, respectively. $N$ and $H$ are time-dependent gating variables for the potassium and hyperpolarizationactivated currents, respectively. The gating variable, $N$, is modified by $\lambda_{N}$, a hyperbolic, U-shaped curve whose nadir height and eccentricity are determined by $\varphi_{N}$, which equals $0.002 \mathrm{~ms}^{-1}$. The variable $\tau_{\mathrm{h}}$ is the voltagedependent recovery time constant. It controls the rate of change of $H$ so that $H$ changes less steeply for more depolarized voltages.

A database of 3600 neurons was constructed and oscillation frequencies were measured. Individual neuron simulations were done with MATLAB's variable time step ode45 integrator with the maximum time step capped at 50 ms. Simulations for each conductance combination were $330 \mathrm{~s}$ long with the first $30 \mathrm{~s}$ discarded.

\section{Hub neuron candidate selection}

From the database of 3600 model neurons, we selected candidate hub neurons $(\boldsymbol{h n})$ with frequencies of $0.5717 \pm$ $0.15 \mathrm{~Hz}$. This frequency was chosen because it is intermediate between the fast and slow half-center oscillation frequencies in the circuit (described below). These coarse tolerance candidates were then used to seed a finer tolerance hub neuron candidate search. For each coarse tolerance candidate, a cloud of 40 random points within a range of $\pm 10 \mathrm{nS}$ of each of their ionic conductances was generated and tested for hub neuron candidacy based on oscillation frequency. Negative conductance values were excluded. The resulting set of 190 fine tolerance hub neuron candidates had an oscillation frequency of 0.5717 $\pm 0.01 \mathrm{~Hz}$. One $\boldsymbol{h} \boldsymbol{n}$ candidate in any pair that was separated by a Euclidean distance of 2 nS or less was elimi- 


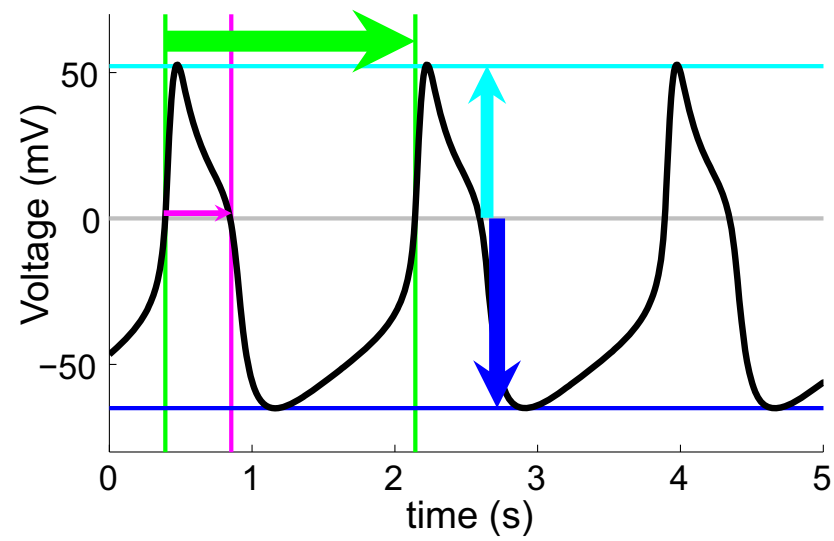

Figure 1 Morris-Lecar model neuron with h-conductance. Voltage wave form example of an oscillating model neuron is shown. In this study, we quantify oscillation frequency (period illustrated by green lines and arrow), duty cycle (magenta), peak voltage (cyan), and baseline voltage (blue).

nated, leaving $143 \boldsymbol{h n}$ candidates that were used for the remaining analysis.

\section{Quantification of individual neuron properties}

The intrinsic properties of the hub neuron candidates were analyzed using our MATLAB code. Duty cycle is defined as the proportion of cycle time that the neuron is above threshold, i.e., the time the oscillator spends at voltages larger than $0 \mathrm{mV}$ divided by the period $(0 \mathrm{mV}$; Fig. 1A, magenta arrow). Here, this quantity was computed as the mean duty cycle of the complete cycles taking place over the last $300 \mathrm{~s}$ of the simulation. The peak and trough voltages are the most positive and most negative voltages in the wave form, respectively (Fig. 1A, cyan and blue lines and arrows). The peak voltage quantity subsequently refers to the mean of the suprathreshold peak voltages over the last $300 \mathrm{~s}$ of simulation time. The trough voltage for each neuron is the mean voltage of the subthreshold troughs over the last $300 \mathrm{~s}$ of the simulation.

Correlations between the various quantities were computed using MATLAB's built-in corrcoef function for obtaining Pearson correlation coefficients and p-values. All correlations were calculated using the entire set of $143 \mathrm{hub}$ neuron candidates. Phase response curves (PRCs) (Gutkin et al., 2001, 2005; Ly et al., 2011) were simulated by giving a $50 \mathrm{~ms}$ current pulse of $\pm 50 \mathrm{nA}$ during various phases of the neuron's cycle and calculating the change in the period due to the current pulse.

\section{Circuit simulations}

The $\boldsymbol{h} \boldsymbol{n}$ candidates were individually placed into the hub position of the five-cell network as described in Gutierrez et al. (2013). Simulations were done with MATLAB's variable time step ode45 integrator. All network simulations were $655 \mathrm{~s}$ long with the first $55 \mathrm{~s}$ discarded. Circuit neuron frequencies were calculated from the inverse of the mean interspike intervals over the last $600 \mathrm{~s}$ of the simulation.
All synapses were modeled as instantaneous. The electrical conductance, $\bar{g}_{e l}$, determined the strength of the electrical synapses.

$$
I_{\text {elec }}=\bar{g}_{e l}\left(V_{m}^{\text {post }}-V_{m}^{\text {pre }}\right)
$$

Chemical inhibitory synapses were modeled by equations modified from Prinz et al. (2004). $S_{\infty}$ is the steadystate synaptic current-gating variable and was modeled after the graded synaptic transmission seen in crustacean stomatogastric neurons (Manor et al. 1997). We used inhibitory synaptic conductance strengths $\left(\bar{g}_{\text {synHC }}\right.$ and $\bar{g}_{\text {syn } 1}$ ) ranging from $0 \mathrm{nS}$ to $10 \mathrm{nS}$. Each of the two synapses that form a half-center coupling have the conductance value denoted by $\bar{g}_{\text {synHC }}$.

$I_{s y n}=\bar{g}_{s y n} S_{\infty}^{\text {pre }}\left(V_{m}^{\text {post }}-V_{s y n}\right), S_{\infty}=1 /\left(1+\exp \left(v_{t h}-\right.\right.$ $\left.V_{m} / v_{\beta}\right)$ ) where $v_{\beta}=5 \mathrm{mV}$ and $v_{\mathrm{th}}=-25 \mathrm{mV}$.

All model neurons in this study are endogenous oscillators and the maximal conductances for the different neurons were chosen to achieve the intrinsic oscillation frequencies required. Conductances for the neurons in the five-cell circuit were as follows [fast; $\boldsymbol{f 1}, \mathbf{f 2}: \bar{g}_{\mathrm{Ca}}=1.9 \times$ $10^{-2} \mu \mathrm{S}, \bar{g}_{K}=3.9 \times 10^{-2} \mu \mathrm{S}, \bar{g}_{h}=2.5 \times 10^{-2} \mu \mathrm{S} ;$ hub neuron (default parameters); $\boldsymbol{h n}$ : $\bar{g}_{C a}=1.7 \times 10^{-2} \mu \mathrm{S}$, $\bar{g}_{K}=1.9 \times 10^{-2} \mu \mathrm{S}, \bar{g}_{h}=8.0 \times 10^{-3} \mu \mathrm{S} ;$ slow; s1,s2: $\bar{g}_{C a}=8.5 \times 10^{-3} \mu \mathrm{S}, \bar{g}_{K}=1.5 \times 10^{-2} \mu \mathrm{S}, \bar{g}_{h}=1.0 \times$ $10^{-2} \mu \mathrm{S}$; all $\left.\bar{g}_{\text {leak }}=1 \times 10^{-4} \mu \mathrm{S}\right]$.

\section{Circuit behavior definitions and tolerances}

Circuit output was quantified using our MATLAB code to determine synchrony among the different neurons within the five-cell circuit. Neurons oscillating within $0.05 \mathrm{~Hz}$ of each other were deemed synchronous. The output pattern of the circuit is classified by which neurons are synchronously active.

\section{Results}

\section{Neuron database}

A database of Morris-Lecar neurons modified by a hyperpolarization-activated current was created (Fig. 2). A coarse sampling of the three-dimensional conductance space formed by the potassium, calcium, and h-conductances yielded many intrinsically oscillating neurons. In Figure 2, we show two-dimensional plots of the effects of altering $\bar{g}_{C a}$ and $\bar{g}_{h}$ on oscillation frequency for each of 15 values of $\bar{g}_{K}$. The regions of white space indicate sets of parameters at which the neuron is not oscillatory. As $\bar{g}_{K}$ is increased, oscillatory behavior becomes increasingly predominant. Note that at each value of $\bar{g}_{K}$ and $\bar{g}_{C a}$, increasing $\bar{g}_{h}$ increases the oscillation frequency. In contrast, increasing $\bar{g}_{C a}$ produces a rotated " $U$ " in oscillator frequency, with the highest frequencies seen at the middle range of $\bar{g}_{C a}$. Consequently, at each value of $\bar{g}_{h}$ and $\bar{g}_{K}$, it is likely that there are two or more models (with different $\bar{g}_{\mathrm{Ca}}$ values) that produce similar frequency oscillations.

We took two neurons with intrinsic frequencies within $1.5 \%$ of each other $(0.0083 \mathrm{~Hz}$ difference) from among model neurons with a maximal potassium conductance of $40 \mathrm{nS}$. In Figure 3A, these are shown with dashed outlines 

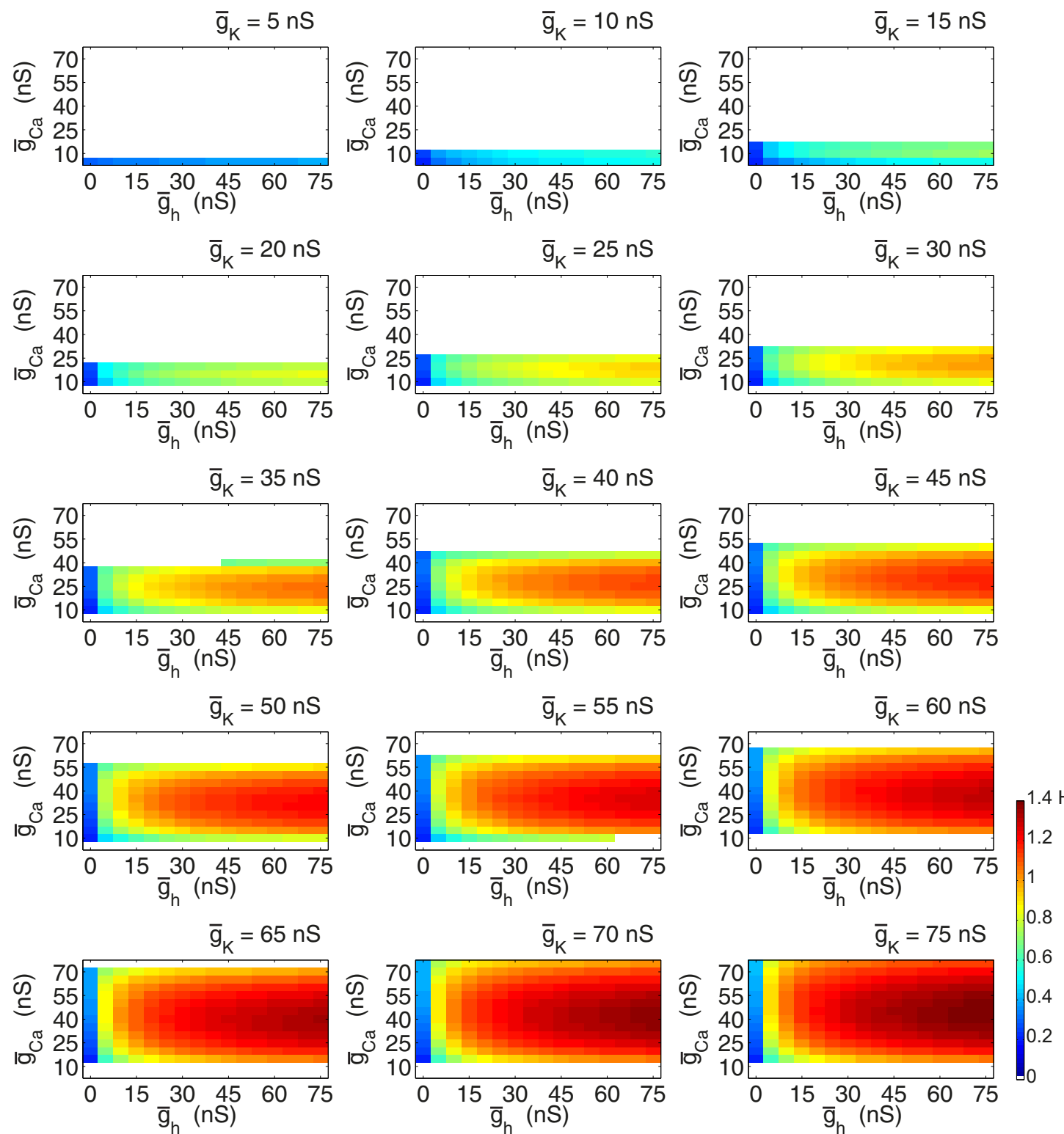

Figure 2 Morris-Lecar neuron with h-conductance database frequencies. This series of panels show the oscillation frequencies of the individual neurons in the database with intrinsic ionic conductances corresponding to the titles and axes. Neuron potassium conductance increases from the top left to bottom right panels. Maximal calcium conductance is on the $y$-axis and maximal $\mathrm{h}$-conductance is on the $x$-axis. The color bar in the bottom right corner conveys the frequency color coding that applies for all plots in this figure. White spaces indicate neurons that are silent, non-oscillatory, or do not cross threshold. All neurons have a $\bar{g}_{\text {leak }}$ of $0.1 \mathrm{nS}$.

at the two sets of parameters that give rise to these neurons. The voltage traces (Fig. 3B) reveal that, although these two neurons have similar frequencies, they have very different peak voltages, amplitudes, and duty cycles. Figure $3 \mathrm{C}$ shows the duty cycles for all of the model neurons with $\bar{g}_{K}=40 \mathrm{nS}$ and indicates that duty cycle in the isolated neurons increases steadily as $\bar{g}_{C a}$ increases. This increase in duty cycle effectively explains the rotated $U$ shape of the frequencies seen in Figure $3 \mathrm{~A}$, because as the plateau phase of the oscillator increases, eventually the oscillator period must extend because the interburst interval has to be long enough to allow the next burst to occur (Skinner et al., 1993, 1994).

The phase response curves (PRCs) of these two neurons further illustrate their differences (Fig. 3D). When positive pulses are delivered at various points in their phases, the neuron with the lower calcium conductance shows a phase advance over most of the cycle. In contrast, the PRC of the higher calcium conductance neuron shows more modest advances late in the cycle. The differences in these PRCs are associated with the length of the action potential depolarization (see voltage traces). 
A Oscillation Frequency
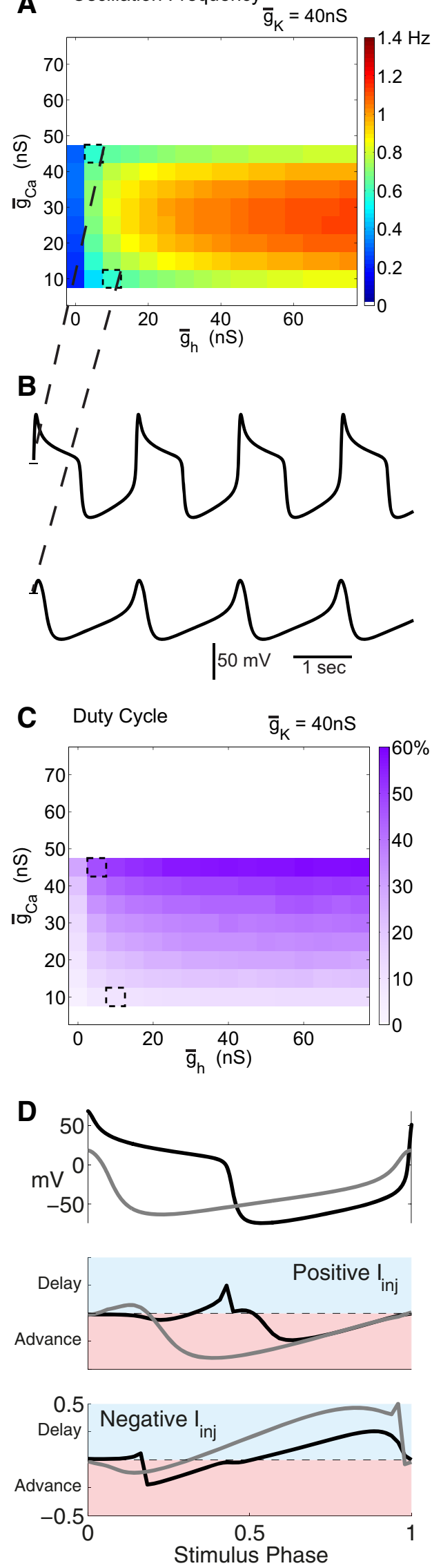

When negative pulses are delivered, both neurons are delayed late in the cycle, with advances seen early in the cycle. Again, this makes sense as the transition between the advance and delay is determined, in part, by the duration of the action potential. A negative current pulse during the plateau terminates the spike early, thus permitting the next spike to occur sooner than without the negative current pulse.

\section{Competing oscillators circuit model}

We next wanted to look at the consequences of altering the intrinsic properties of an individual neuron on a circuit's performance. For this purpose, we made use of a circuit model of intrinsic oscillators that compete for the recruitment of a hub neuron (hn; Fig. 4A) (Gutierrez et al., 2013). The competing components of the circuit are two sets of mutually inhibitory half-center oscillators, one fast and one slow set. $\boldsymbol{f} \mathbf{1}$ and $\mathbf{f} \mathbf{2}$ are identical, intrinsically fast, oscillating neurons whose mutually inhibiting synapses have conductance strength $\bar{g}_{s y n H C}$. Likewise for $s 1$ and $\boldsymbol{s 2}$, which are intrinsically slow oscillators. The $\boldsymbol{h n}$ is electrically coupled $\left(\bar{g}_{\text {el }}\right)$ to one neuron in each half-center pair and it receives an inhibitory synapse $\left(\bar{g}_{\text {syn } 1}\right)$ from the other neuron in each half-center pair. Without $\bar{g}_{e l}$ and $\bar{g}_{s y n}, \boldsymbol{h n}$ oscillates at a frequency that is directly between that of the fast and slow half-center frequencies. The frequencies of all of the neurons in the circuit can be displayed at once using a set of colored concentric circles and squares, as illustrated in the top right of Figure $4 \mathrm{~A}$ and above the traces in Figures 4, B and C.

The circuit produces a number of different output patterns depending on the synaptic conductances chosen. For example, in Figure 4B, the fast half-center pair is oscillating at a high frequency (top traces and the two outer orange circles depicting the $\boldsymbol{f} \mathbf{1}$ and $\mathbf{f} \mathbf{2}$ frequencies) relative to the slow half-center pair (bottom traces and the inner blue circles depicting the $\boldsymbol{s} \mathbf{1}$ and $\boldsymbol{s} 2$ frequencies). In this instance, the electrical coupling is low while the synaptic inhibition onto $\boldsymbol{h} \boldsymbol{n}$ is high and $\boldsymbol{h n}$ oscillates at the frequency of the slow oscillators (Fig. 4B; middle trace and blue square nested between orange circles and blue circles). In Figure 4C, the electrical coupling is high while synaptic inhibition to $\boldsymbol{h} \boldsymbol{n}$ is low and all neurons in the circuit oscillate at the same frequency (as shown in the

Figure 3 Comparison of two database neurons. A, A selected panel from the neuron database is displayed in which $\mathrm{K}^{+}$conductance is $40 \mathrm{nS}$. Two neurons in this plane have frequencies very close to each other despite their disparate ionic conductances $\left[\bar{g}_{h}=5 \mathrm{nS}, \bar{g}_{\mathrm{Ca}}=45 \mathrm{nS}, 0.5705 \mathrm{~Hz} ; \bar{g}_{h}=10 \mathrm{nS}, \bar{g}_{\mathrm{Ca}}=10\right.$ $\mathrm{nS}, 0.5787 \mathrm{~Hz}$. $\boldsymbol{B}$, These two neurons differ in their wave form profile, however, as shown in the traces. C, A plot of duty cycles illustrates how much these two neurons differ in that respect. Duty cycle is depicted as the percentage of the cycle in which the neuron is above threshold. $\boldsymbol{D}$, Phase response curves. One full period is plotted (top) for each of the two neurons highlighted above. The neuron with the higher maximal calcium conductance is in black and the lower calcium neuron is shown in gray. PRCs in response to positive current pulses of $50 \mathrm{nA}$ (middle) and PRCs in response to negative current pulses of $-50 \mathrm{nA}$ (bottom) are shown. 
A

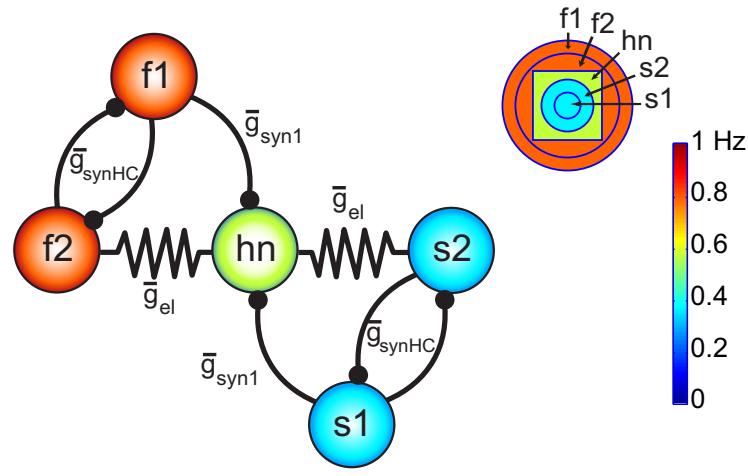

B

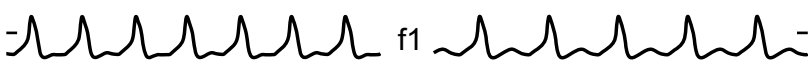

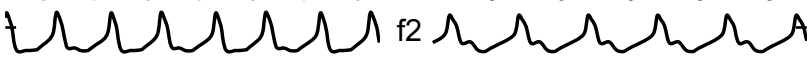
- rorsinns - n

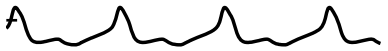
s1 $\overbrace{100 \mathrm{mV}}$

D

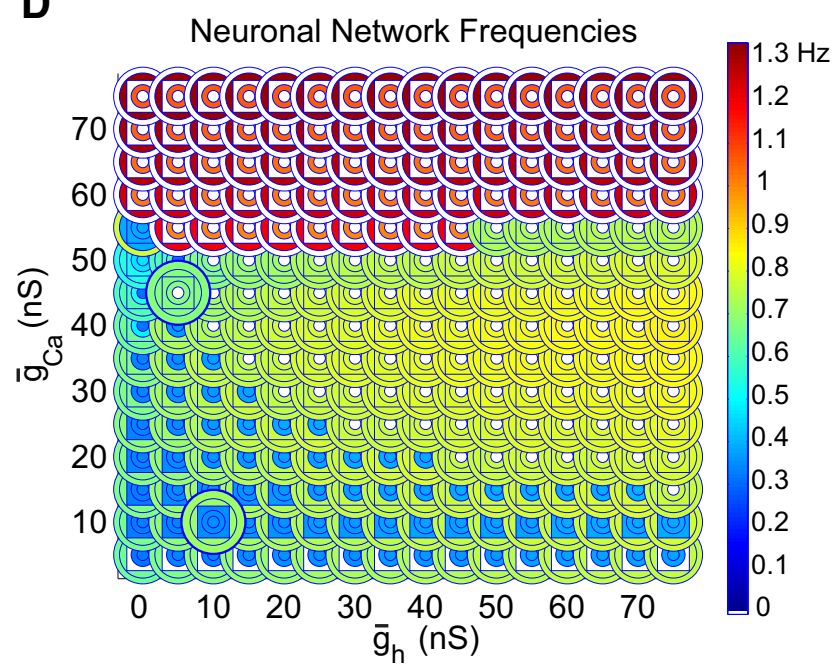

E

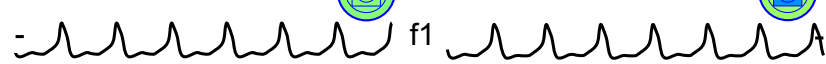

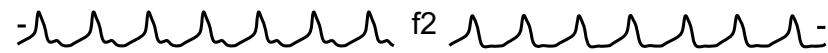
oururann nuruss r r

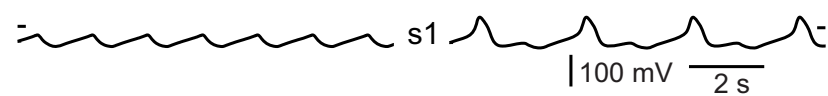

Figure 4 Model circuit. $\boldsymbol{A}$, The model circuit in this study contains five model neurons with distinct intrinsic properties. The $\boldsymbol{f 1}$ and f2 neurons are the intrinsically fast oscillating neurons in the circuit while the $\mathbf{s} 1$ and $\mathbf{s} 2$ neurons are intrinsically slowly oscillating. The $\boldsymbol{h} n$ 's intrinsic frequency is intermediate of the fast and slow half-center frequencies. The key above and to the right of the circuit introduces a parameterscape point. The series of concentric shapes relay the frequencies of the neurons in the circuit with $\mathbf{s} 1$ represented by the innermost circle, $\mathbf{f 1}$ repre- traces and the five green concentric shapes above them). Previous work using this circuit (Gutierrez et al., 2013) demonstrated that modulation of inhibitory and electrical synaptic conductances can alter circuit behavior, as shown in this example. In contrast, the present study explores the modulation of ionic conductances in the $\boldsymbol{h n}$ and the subsequent effect on circuit output.

\section{Hub neuron intrinsic conductances shape circuit behavior}

Using the five-cell circuit model, we studied the effect on circuit output when synaptic conductances remain constant and only the ionic conductances of the $\boldsymbol{h n}$ are changed. In Figure 4D, we observe the circuit output as a function of two of the $\boldsymbol{h} \boldsymbol{n}$ ionic conductances; $\bar{g}_{C a}$ and $\bar{g}_{h}$. Essentially, each of the database neurons from Figure $3 \mathrm{~A}$ is inserted into the circuit in the $\boldsymbol{h} \boldsymbol{n}$ position. At each point of this "parameterscape" (Gutierrez et al., 2013), the behavior of the full circuit is indicated as a set of nested, color-coded circles and squares that are colored according to the frequency exhibited by each circuit neuron (Fig. 4D). Note that some neurons that were not oscillatory in isolation do oscillate when placed into the circuit as the $\boldsymbol{h n}$. This is the case for neurons with a $\bar{g}_{\mathrm{Ca}}$ value of $50 \mathrm{nS}$ and a few neurons with a $\bar{g}_{\mathrm{Ca}}$ of $55 \mathrm{nS}$. More importantly, Figure 4D shows that when the two neurons indicated in the boxes in Figure 3A are placed in the network as the $\boldsymbol{h n}$ with all other circuit parameters being identical, the circuit outputs are entirely different despite the two neurons having almost identical frequencies when in isolation. In one case (the $\boldsymbol{h n}$ with high $\bar{g}_{\mathrm{Ca}}$ ), four of the neurons oscillate at a moderate frequency and $\mathbf{s 1}$ is silent (Fig. 4E). In the other case (the $\boldsymbol{h n}$ with low $\bar{g}_{\mathrm{Ca}}$ ), all five neurons are oscillatory, but $\mathbf{f 1}$ and $\mathbf{f 2}$ oscillate at a moderate frequency, while the other three neurons oscillate together at a low frequency (Fig. 4F).

sented by the outermost circle, and $\boldsymbol{h n}$ represented by the square between the circles for $\mathbf{f 2}$ and $\mathbf{s} 2$. The colors of these shapes convey the neuron's oscillation frequency according to the color bar heat map. This particular point (above circuit, right) conveys the frequencies of the five neurons when $\boldsymbol{h n}$ is not electrically coupled nor synaptically inhibited by the other neurons in the circuit. $\boldsymbol{B}$, When the electrical coupling conductance $\left(\bar{g}_{e l}\right)$ is $2 \mathrm{nS}$ and the synaptic inhibition to $\boldsymbol{h n}\left(\bar{g}_{\text {syn } 1}\right)$ is $6 \mathrm{nS}$, the circuit exhibits a behavior in which $\boldsymbol{h n}$ oscillates in time with the slow oscillators as illustrated by the traces and the parameterscape point $\left(\bar{g}_{\text {synHC }}\right.$ is $\left.5 \mathrm{nS}\right)$. C , When $\bar{g}_{e l}$ is $6 \mathrm{nS}$ and $\bar{g}_{\text {syn } 1}$ is $2 \mathrm{nS}$, the circuit exhibits a behavior in which all of the neurons oscillate at the same frequency. $\boldsymbol{D}$, The neurons from Figure $3 A$ are inserted into the five-cell circuit as the hub neuron. This parameterscape illustrates the circuit behavior that results from having any of these model neurons take their place as the hub neuron. The concentric shapes indicate the frequencies of the circuit neurons according to the key $(\boldsymbol{A})$. The two parameterscape points that are popped-out correspond to the two dashed boxes Figure $3 A$. Circuit synaptic parameters for all points in the parameterscape are as follows; $\bar{g}_{\text {syn } 1}=3 \mathrm{nS}, \bar{g}_{\text {synHC }}=5 \mathrm{nS}, \bar{g}_{\mathrm{el}}=$ $3 \mathrm{nS}$. $\boldsymbol{E}, \boldsymbol{F}$, Voltages traces display the circuit activity at the two highlighted points. 
A

Duty Cycle

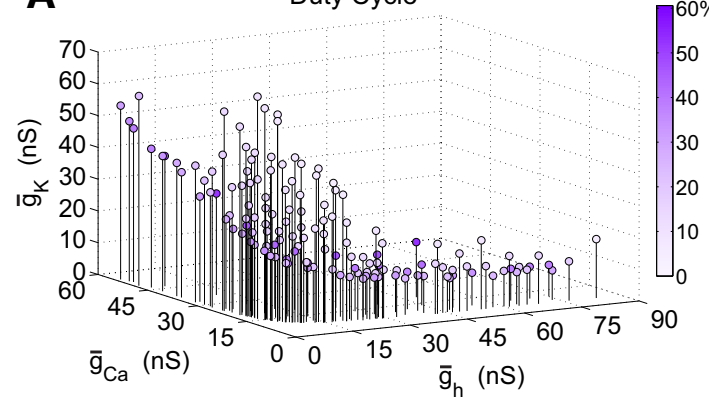

B

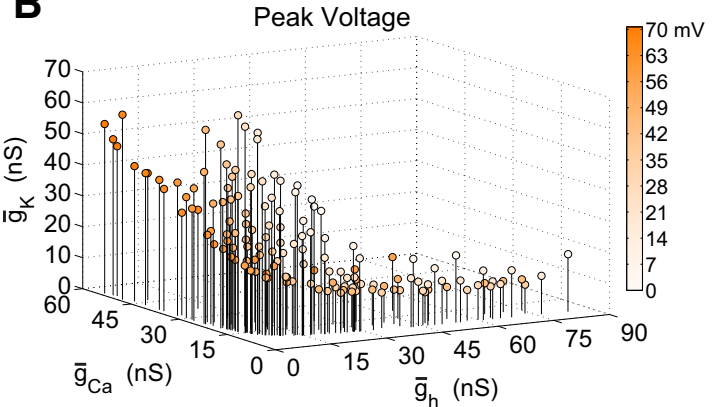

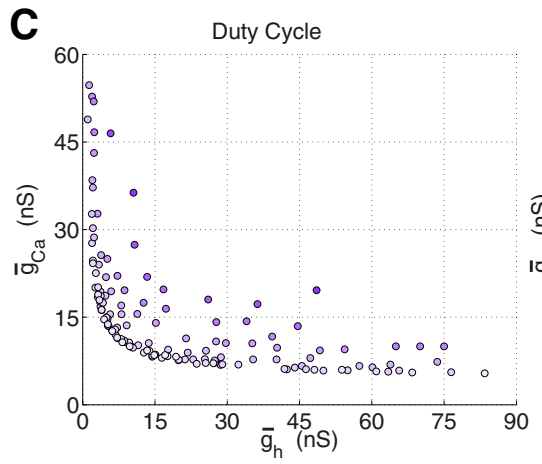
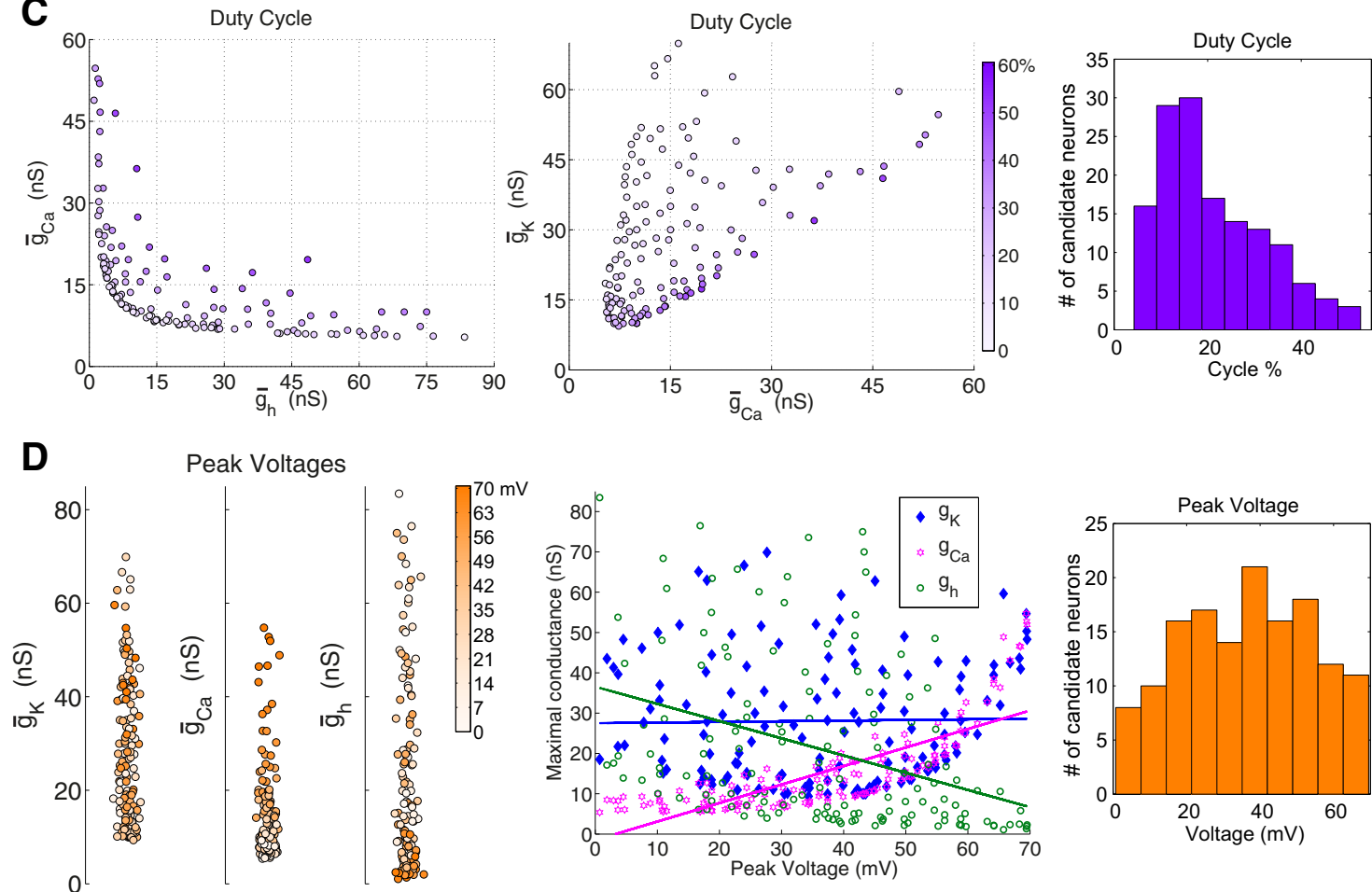

Figure $\mathbf{5} \boldsymbol{h} \boldsymbol{n}$ candidates and intrinsic properties. The $\boldsymbol{h} \boldsymbol{n}$ candidates all have isolated frequencies within the range of $0.5717 \pm 0.01$ $\mathrm{Hz}$. The set of 143 individual neurons spans ionic conductance space in a V-formation. $\boldsymbol{A}, \boldsymbol{h} \boldsymbol{n}$ candidate duty cycles spanned the range of $4.27 \%$ to $52.7 \%$. B , The peak voltages are the mean supra-threshold oscillation peak voltages and these ranged from 0.7132 to $69.5211 \mathrm{mV}$. C, The first two panels are two-dimensional plots of duty cycles containing the same data as $\boldsymbol{A}$. A histogram for $\boldsymbol{h} \boldsymbol{n}$ candidate duty cycles (right); mean duty cycle is $21.33 \%$, standard deviation 11.24. $\boldsymbol{D}$, One-dimensional plots for peak voltages as function of individual $\boldsymbol{h} \boldsymbol{n}$ conductances (left) make the correlations in $\boldsymbol{B}$ more apparent. These are further summarized in the middle plot showing the relationships between the three conductances and the peak voltages of the $\boldsymbol{h} \boldsymbol{n}$ candidates with regression lines. Right, Histogram for $\boldsymbol{h} \boldsymbol{n}$ candidate peak voltages; mean of peak voltages is $36.2955 \mathrm{mV}$, standard deviation $17.8493 \mathrm{mV}$.

The differences between the waveforms for the two neurons shown in Figure 3B suggest an explanation for the different circuit behaviors that are produced in Figure 4, D-F. When the $\boldsymbol{h} \boldsymbol{n}$ has a long duration, large amplitude oscillation, the electrical coupling is sufficient to synchronize the $\mathbf{f 2}, \boldsymbol{h n}$, and $\mathbf{s} \mathbf{2}$ neurons, and the $\mathbf{f 1 / f 2}$ half-center maintains its alternation. The $\mathbf{s} 1$ neuron doesn't have enough $\mathrm{h}$-conductance to rebound from the relatively rapid $\mathbf{s} 2$ inhibitions it receives, and thus it falls silent. In the second case (low $\boldsymbol{h} \boldsymbol{n} \bar{g}_{\mathrm{C}}$ levels), the electrical coupling synchronizes the bursts in $\mathbf{f 2}, \boldsymbol{h n}$, and $\mathbf{s} \mathbf{2}$, but the inhibition from $\mathbf{s 1}$ (which alternates with s2) is strong enough to suppress the $\boldsymbol{h} \boldsymbol{n}$ whose oscillations barely cross threshold. Therefore it only fires every other fast cycle, thus joining the slow oscillatory regime.

\section{Intrinsic properties of hub neuron candidates with same frequency}

We created a population of model neurons with vastly ranging conductances and similar frequencies to examine the effects of modulation of the intrinsic properties of these centralized neurons on circuit output. Therefore, we generated 143 hub neuron candidates with frequencies of $0.5717 \pm 0.01 \mathrm{~Hz}$. Figure 5 shows their properties.

The resulting $143 \boldsymbol{h n}$ candidates span ionic conductance space in an L-shape that hugs the $\bar{g}_{C a}$ and $\bar{g}_{h}$ axes, 
indicating that the two inward currents compensate for each other to some degree (Fig. 5). Indeed, the three conductances are all significantly correlated with each other $(p<0.05)$. As one might expect, there is a positive correlation between the $\mathrm{Ca}^{2+}$ and $\mathrm{K}^{+}$conductances $(r=$ 0.48 ). However, the h-conductance is negatively correlated with the $\mathrm{Ca}^{2+}$ and $\mathrm{K}^{+}$conductances $(r=-0.68$ and -0.53 , respectively).

The duty cycles in this group of $\boldsymbol{h} \boldsymbol{n}$ candidates spanned a broad range (Fig. 5A,C). The $\boldsymbol{h} \boldsymbol{n}$ candidates along the inner rim of the L-formation tend to have longer duty cycles than the candidates outside of the elbow. The $\mathrm{Ca}^{2+}$ and h-conductances are positively correlated with duty cycle but only the $\mathrm{Ca}^{2+}$ conductance is significant $(r=0.46, p<0.001 ; r=0.14, p=0.085)$. The $\mathrm{K}^{+}$ conductance is negatively and significantly correlated with duty cycle $(r=-0.40, p<0.001)$.

The voltage excursions of the candidate neurons' oscillations also vary considerably. The maximum voltages of the oscillation peaks are as high as $+70 \mathrm{mV}$ for some neurons, but barely cross threshold in others (Fig. 5B,D). Voltage minima (not shown) are less variable, with a mean of $-61.9 \mathrm{mV}$ and a standard deviation of $8.0 \mathrm{mV}$. All conductances are significantly correlated with baseline voltages as well as the full voltage range. The correlation between peak voltage and either $\mathrm{Ca}^{2+}$ or $\mathrm{h}$-conductance is highly significant $(p<0.001)$, but there is no significant correlation between $\mathrm{K}^{+}$conductance and peak voltage $(p$ $=0.82) . \mathrm{Ca}^{2+}$ conductance is positively correlated with peak voltage $(r=0.78)$, whereas h-conductance is negatively correlated with it $(r=-0.37)$ (Fig. 5D, left and middle). The relationship between $\mathrm{Ca}^{2+}$ conductance and peak voltage can be seen in the distribution of peak voltage values in the conductance space as candidate hub neurons with higher $\mathrm{Ca}^{2+}$ conductances also tend to have higher peak voltages (Fig. 5B and D, left). Likewise, the negative correlation between $\mathrm{h}$-conductance and peak voltage is seen in the decreasing peak voltages for increasing $\mathrm{h}$-conductance.

\section{Impact of the hub neuron's intrinsic conductances on circuit output}

We next embedded each of the 143 candidate $\boldsymbol{h} \boldsymbol{n}$ neurons (from Fig. 5) into two different versions of the five-cell circuit, one with a high value for $\bar{g}_{s y n 1}$ and low $\bar{g}_{e l}$, and vice versa for the other. This allowed us to determine how each of a large set of neurons with the same frequency in isolation influences circuit output. By studying two sets of synaptic circuit parameters, we were also able to observe the extent of the synaptic parameters' influence on the circuit output. For the sake of tractability, the maximal ionic conductances of the intrinsically slow and fast oscillator neurons remained unchanged.

In Figure 6, the resulting circuit output is shown for the two different circuit configurations. With high $\bar{g}_{\text {syn } 1}$ and low $\bar{g}_{e l}$ (Fig. 6A), almost all of the $\boldsymbol{h} \boldsymbol{n}$ candidate-containing circuits produced qualitatively similar behavior. This behavior is characterized by $\boldsymbol{h} \boldsymbol{n}$ being active with the slow half-center oscillators while the fast half-centers oscillate at their own frequency (output pattern indicated in the legend by dark blue color). Although the network pattern is largely the same for this synaptic configuration, these networks do not necessarily have identical frequencies. The networks with higher overall frequencies generally had hns with lower $\mathrm{Ca}^{2+}$ and $\mathrm{K}^{+}$conductances and higher h-conductances (data not shown). With the synaptic conductance values inverted so that $\bar{g}_{s y n}$ is low and $\bar{g}_{e l}$ is high, a number of qualitatively different circuit behaviors are seen for the different $\boldsymbol{h} \boldsymbol{n}$ neurons (Fig. 6B). Several of them produce the behavior represented by dark blue in which $\boldsymbol{h} \boldsymbol{n}$ is active with the slow oscillators, especially when $\boldsymbol{h} \boldsymbol{n}$ has a relatively high $\mathrm{h}$-conductance. Unlike in Figure 6A, Figure 6B displays a variety of other circuit output patterns. Clustered around low values of $\bar{g}_{h}$ are circuits in which $\boldsymbol{h} \boldsymbol{n}$ joins the fast oscillators (represented by red). Also for low values of $\bar{g}_{h}$ but spanning the range of $\bar{g}_{C a}$, there are circuits in which all but the $\mathbf{s 1}$ neuron are part of the fast oscillator (purple). There are also a few circuits in which all five neurons are active at the same frequency (green). These tend to have low values of all three ionic conductances. Finally, there are circuits (white diamonds) that were sufficiently irregular to defy simple classification. Such circuits tend to lie at the boundaries between clusters of circuits with classifiable behavior.

For the synaptic configuration with low electrical coupling and high synaptic coupling in Figure 6A, it is not simply a matter of having sufficiently weak electrical coupling to allow the networks to be in an inhibitiondominated regime because with still lower electrical coupling $\left(\bar{g}_{\mathrm{el}}=0.5 \mathrm{nS}\right)$, the candidate circuits produce a variety of network patterns. This is an effect of the electrical coupling being too weak to produce integer coupling among the distinct rhythms in the circuit (Gutierrez et al., 2013). Nor is the configuration in Figure $6 \mathrm{~A}$ dependent on a precisely tuned value of the electrical coupling. This same effect is observed with electrical coupling up to $4 \mathrm{nS}$ (with all other parameters kept the same) as well as with a number of different circuit configurations. Therefore, these particular examples in Figure 6 contrast two qualitatively different regimes. In one regime, modulation of the hub neuron results in significant alteration of the circuit patterns. In contrast, in the other regime, modulation of the hub neuron produces only modest circuit changes, which ultimately do not lead to a change in the qualitative behavior of the network.

\section{Discussion}

All nervous systems are modulated, both by hormones and local modulatory inputs (Marder, 2012). Neuromodulatory substances such as amines and neuropeptides can modify one or more voltage-dependent conductances (Levitan, 1988; Nicoll, 1988; McCormick, 1989), can act directly on signal transduction pathways (Levitan and Levitan, 1988; Clark et al., 2008; Zhang et al., 2010; Shinkai et al., 2011), and can result in changes in synaptic transmission (Kandel and Schwartz, 1982; Thirumalai et al., 2006; Nadim and Bucher, 2014).

In the stomatogastric ganglion, one of the most intensively studied small circuits, every neuron and every synapse is subject to neuromodulation by one or more 

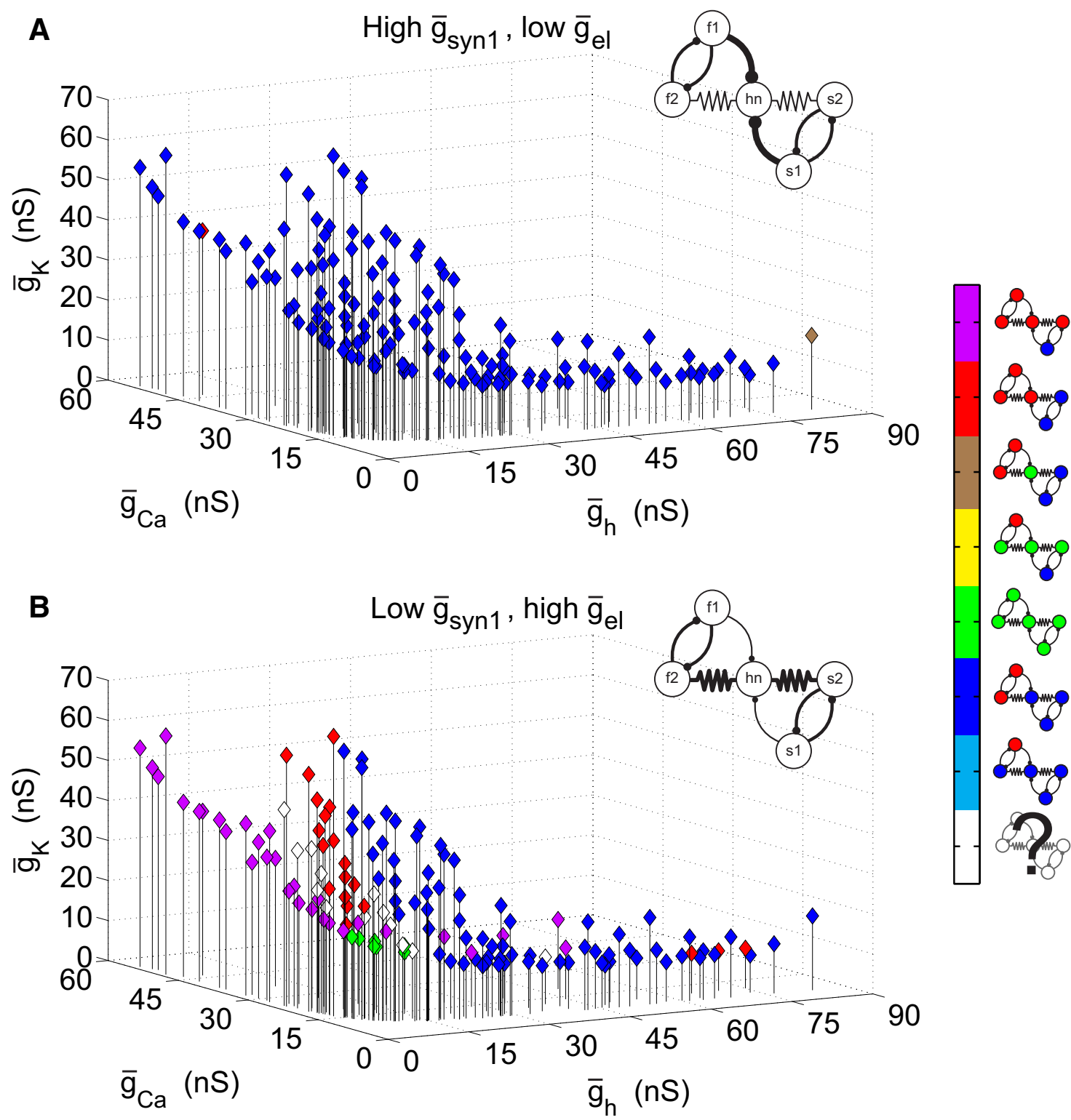

Figure 6 Circuit behavior with different hub neurons. Each diamond represents a circuit that contains an $\boldsymbol{h n}$ candidate with intrinsic conductances at that particular point in ionic conductance space. The circuit behavior that results is encoded according to the color key on the right. $\boldsymbol{A}, \bar{g}_{s y n 1}=6 \mathrm{nS}, \bar{g}_{e l}=2 \mathrm{nS}$; most of the $\boldsymbol{h} \boldsymbol{n}$ candidate-containing circuits with these synaptic parameters display a behavior in which $\boldsymbol{h} \boldsymbol{n}$ is active with the slow oscillators while the fast oscillators are active at their own frequency. $\boldsymbol{B}, \bar{g}_{\text {syn } 1}=2$ nS, $\bar{g}_{\text {el }}=6 \mathrm{nS}$; with these synaptic parameters, there is much more variability among the output from $\boldsymbol{h n}$ candidate-containing circuits. $\bar{g}_{\text {synHC }}$ is $5 \mathrm{nS}$ for both panels.

substances (Marder and Bucher, 2007; Stein, 2009; Harris-Warrick, 2011; Johnson et al., 2011; Marder et al., 2014; Nadim and Bucher, 2014). Additionally, some neuromodulatory actions are contingent on the presence of other modulators, or recent history of neuromodulation, often with significant behavioral consequences (Dickinson et al., 1997; Nadim et al., 2008; Chalasani et al., 2010; Wu et al., 2010; Rodgers et al., 2011a, 2011b; Jang et al., 2012; Kwiatkowski et al., 2013; Krenz et al., 2014; Nadim and Bucher, 2014). In principle, the action of any given neuromodulator on a circuit can be restricted to a few circuit elements, or can be more widespread. How a given neuromodulator reconfigures a specific circuit depends on which neurons are direct targets of the modulator (Swensen and Marder, 2001), and secondarily on how the affected or modulated neuron interacts with other circuit components (Nadim and Bucher, 2014). In this paper, we restrict ourselves to "modulation" of a single neuron, and determine its influence, or lack thereof, on an entire circuit. Perhaps remarkably, there is a richness of behaviors to be seen when only one neuron is modulated. But, in principle, one could investigate the consequences of modulating multiple targets in different circuit configurations. 
Our results are in line with previous results that have shown that the synchronization properties of a network can change when the properties of a single neuron are varied but its frequency in isolation is maintained (Ermentrout et al., 2001). In this study, we used parameter sweeps of the three active conductances in the hub neuron to model the changes in intrinsic membrane properties that could occur during neuromodulation or homeostasis. Of course, biological neurons often have many more conductances, any and all of which are likely to be subject to modulation. We chose to use a relatively simple model rather than one with many more conductances because the three conductances that we modeled capture the basic dynamics seen in most neurons and they each have distinct functions. Thus, the relative simplicity of the model used here gives results that are more directly visible and visualizable than is often the case with more complex models. Conversely, we chose a conductance-based model over a more simple phaseoscillator model so as to relate our results to the actions of specific currents (i.e., depolarizing, hyperpolarizing, or hyperpolarization-activated depolarization). Although we have chosen to highlight a few specific cases in this very general model, our findings address the possible consequences of targeted modulation for oscillatory circuits with electrical and inhibitory chemical synapses-such as those one might find in any invertebrate or vertebrate nervous system.

\section{Characterization of the properties of the Morris-Lecar model with $\mathrm{h}$-conductance}

Altering the conductances of the three currents in the hub neuron model produces substantial changes in the frequency, wave form, duty cycle, and the amplitudes of the voltage excursions of this model neuron. Increasing the $\mathrm{Ca}^{2+}$ conductance produces monotonic increases in duty cycle (Fig. 3), which accounts for the U-shaped effect of increasing the $\mathrm{Ca}^{2+}$ conductance on frequency. This occurs because the oscillator continuously broadens, which eventually forces it to decrease in frequency. This is similar to behavior in the unmodified Morris-Lecar (Skinner et al., 1993, 1994), and demonstrates that even very simple model neurons can show quite different behaviors as a function of parameter choices. Of course, this model does not generate fast action potentials, but rather slower oscillations, on which action potentials could ride if they had also been modeled. Given the increased use of methods that monitor $\mathrm{Ca}^{2+}$ dynamics as a proxy for voltage in many invertebrate and vertebrate preparations, the fact that an increase in frequency could occur as a function of either increasing or decreasing calcium conductance is important to know for the potential interpretation of either genetic or pharmacological manipulations of currents.

Other studies have used conventional Morris-Lecar models (Wang and Rinzel, 1992; Skinner et al., 1993, 1994; Rinzel and Ermentrout, 1998; Tsumoto et al., 2006; Zhang et al., 2009). The Morris-Lecar with h-current (LoFaro et al., 1994) has useful features that make it a valuable tool for studying neuron and circuit dynamics. It is simple, yet captures more physiological detail than the conventional Morris-Lecar model. The h-current expands the voltage range and produces more realistic responses to inhibition, especially when the modeled system itself has an h-current. Therefore, for circuits with inhibition, it may be preferable to other reduced models. Additionally, the modified Morris-Lecar, as well as the conventional Morris-Lecar-or any other conductance based oscillator-allows one to attribute neuronal and network dynamics to physiologically based currents.

\section{Global versus local changes}

One of the big challenges in understanding how neuromodulators change the output of neuronal circuits is determining which changes in circuit performance arise from the direct actions of a modulator on individual target neurons, without appreciably changing the behavior of the other circuit elements, and which emerge as a consequence of the modulated neuron(s) interacting with other circuit components, thus indirectly altering the activity of many neurons that are themselves not direct targets of the modulator. Figure 6 highlights two extreme cases. In Figure 6A, modulation of the hub neuron will produce local changes, that is, the modulated neuron's intrinsic properties will be altered, and while other neurons in the network may also change their behaviors somewhat because of the electrical coupling, the overall structure of the circuit output is preserved. In contrast, in Figure 6B, changes in only the hub neuron produce global changes, and the coordination patterns among all of the circuit neurons are substantially altered, merely by changing the properties of a single neuron.

Importantly, the two cases have identical connectomes - the synaptic architecture is the same. The intrinsic properties of the neurons are identical in the two cases, and they differ only in the strengths of the chemical and electrical synapses. This work shows that under some sets of circumstances, even modest modulation of one current in one neuron can create a number of different circuit output patterns (Fig. 6B). For example, increasing $\bar{g}_{C a}$ can transition the circuit through three different output patterns. Starting at a point in the elbow of the group where calcium and potassium conductances are low, and increasing those conductance together, as might happen with homeostasis, leads to different output patterns. The circuit would go from a pattern in which the hub neuron is oscillating in time with the slow oscillators, to a pattern in which the hub neuron would switch to oscillating with the fast rhythm, and finally switching to a pattern in which the electrically coupled slow oscillator is recruited into the fast rhythm.

\section{Implications for evaluating connectomes}

It is now widely appreciated that the connectome, while necessary to understand circuit dynamics, is not sufficient (Brezina, 2010; Bargmann, 2012; Bargmann and Marder, 2013), partially because of neuromodulation, and partially as a simple function of the necessity of knowing the intrinsic properties of neurons and the synaptic strengths in the circuit. That said, the ambiguities inherent in the connectome are even more considerable when electrical synapses are part of the circuit, as current can flow 
bi-directionally between neurons and produce changes in circuit output that are difficult to predict, with or without rectification of the electrical synapses (Kopell et al., 1998; Soto-Trevino et al., 2005; Gutierrez and Marder, 2013; Gutierrez et al., 2013).

It is interesting that the limitations of the connectome itself for predicting circuit output also depend on the circuit parameters. In Figure 6B, we see that altering the intrinsic properties of the hub neuron can drastically alter the circuit's behavior. Nonetheless, in other parameter regimes, the circuit is robust to the changes in the intrinsic properties of the hub neuron. In these conditions, an anatomic wiring diagram or "connectome" would adequately describe the circuit's function (Plaza et al., 2014). In other words, a modulatory environment that sets circuit parameters can produce a mode switch from one in which the circuit is insensitive to parameter changes (e.g., well characterized by its connectome), and another in which the entire modulatory environment must be specified, and the connectome sets the stage on which the modulators act to configure the circuit's output. It is now widely appreciated that an anatomic connectome is not necessarily sufficient for understanding how a circuit will work (Bargmann, 2012), but this study shows that the sufficiency of the connectome for elucidating circuit function is in itself under neuromodulatory control.

\section{References}

Bargmann Cl (2012) Beyond the connectome: how neuromodulators shape neural circuits. Bioessays 34:458-465. CrossRef Medline

Bargmann Cl, Marder E (2013) From the connectome to brain function. Nat Methods 10:483-490. Medline

Blitz DM, Nusbaum MP (2012) Modulation of circuit feedback specifies motor circuit output. J Neurosci 32:9182-9193. CrossRef Medline

Brezina V (2010) Beyond the wiring diagram: signalling through complex neuromodulator networks. Philos Trans R Soc Lond B Biol Sci 365:2363-2374. CrossRef Medline

Chalasani SH, Kato S, Albrecht DR, Nakagawa T, Abbott LF, Bargmann Cl (2010) Neuropeptide feedback modifies odor-evoked dynamics in caenorhabditis elegans olfactory neurons. Nat Neurosci 13:615-621. CrossRef Medline

Clark MC, Khan R, Baro DJ (2008) Crustacean dopamine receptors: localization and $\mathrm{G}$ protein coupling in the stomatogastric ganglion. J Neurochem 104:1006-1019. CrossRef Medline

Dickinson PS, Fairfield WP, Hetling JR, Hauptman J (1997) Neurotransmitter interactions in the stomatogastric system of the spiny lobster: one peptide alters the response of a central pattern generator to a second peptide. J Neurophysiol 77:599-610. Medline

Diehl F, White RS, Stein W, Nusbaum MP (2013) motor circuitspecific burst patterns drive different muscle and behavior patterns. J Neurosci 33:12013-12029. CrossRef Medline

Ermentrout B, Pascal M, Gutkin B (2001) The effects of spike frequency adaptation and negative feedback on the synchronization of neural oscillators. Neural Comput 13:1285-1310. Medline

Gutierrez GJ, Marder E (2013) Rectifying electrical synapses can affect the influence of synaptic modulation on output pattern robustness. J Neurosci 33:13238-13248. CrossRef Medline

Gutierrez GJ, O'Leary T, Marder E (2013) Multiple mechanisms switch an electrically coupled, synaptically inhibited neuron between competing rhythmic oscillators. Neuron 77:845-858. CrossRef Medline

Gutkin BS, Laing CR, Colby CL, Chow CC, Ermentrout GB (2001) Turning on and off with excitation: the role of spike-timing asyn- chrony and synchrony in sustained neural activity. J Comput Neurosci 11:121-134. Medline

Gutkin BS, Ermentrout GB, Reyes AD (2005) Phase-response curves give the responses of neurons to transient inputs. J Neurophysiol 94:1623-1635. CrossRef Medline

Harris-Warrick RM (2011) Neuromodulation and flexibility in Central Pattern Generator networks. Curr Opin Neurobiol.

Harris-Warrick RM, Johnson BR (2010) Checks and balances in neuromodulation. Front Behav Neurosci 4:). CrossRef Medline

Harris-Warrick RM, Johnson BR, Peck JH, Kloppenburg P, Ayali A, Skarbinski J (1998) Distributed effects of dopamine modulation in the crustacean pyloric network. Ann N Y Acad Sci 860:155-167. Medline

Jang H, Kim K, Neal SJ, Macosko E, Kim D, Butcher RA, Zeiger DM, Bargmann Cl, Sengupta P (2012) Neuromodulatory state and sex specify alternative behaviors through antagonistic synaptic pathways in C. elegans. Neuron 75:585-592.CrossRef

Johnson BR, Brown JM, Kvarta MD, Lu JY, Schneider LR, Nadim F, Harris-Warrick RM (2011) Differential modulation of synaptic strength and timing regulate synaptic efficacy in a motor network. J Neurophysiol 105:293-304. CrossRef Medline

Kandel ER, Schwartz JH (1982) Molecular biology of learning: modulation of transmitter release. Science 218:433-443. Medline

Kopell N, Abbott LF, Soto-Trevino C (1998) On the behavior of a neural oscillator electrically coupled to a bistable element. Physica D 121:367-395.CrossRef

Krenz WD, Parker AR, Rodgers EW, Baro DJ (2014) Dopaminergic tone persistently regulates voltage-gated ion current densities through the D1R-PKA axis, RNA polymerase II transcription, RNAi, mTORC1, and translation. Front Cell Neurosci 8:39CrossRef Medline

Kwiatkowski MA, Gabranski ER, Huber KE, Chapline MC, Christie AE, Dickinson PS (2013) Coordination of distinct but interacting rhythmic motor programs by a modulatory projection neuron using different co-transmitters in different ganglia. J Exp Biol 216:18271836. CrossRef Medline

Levitan IB (1978) Modulation of neuronal activity by peptides and neurotransmitters: possible role of cyclic nucleotides. J Physiol 74:521-525. Medline

Levitan IB (1988) Modulation of ion channels in neurons and other cells. Annu Rev Neurosci 11:119-136. CrossRef Medline

Levitan ES, Levitan IB (1988) Serotonin acting via cyclic AMP enhances both the hyperpolarizing and depolarizing phases of bursting pacemaker activity in the aplysia neuron R15. J Neurosci 8:1152-1161. Medline

LoFaro T, Kopell N, Marder E, Hooper SL (1994) Subharmonic coordination in networks of neurons with slow conductances. Neural Comp 6:69-84.CrossRef

Ly C, Melman T, Barth AL, Ermentrout GB (2011) Phase-resetting curve determines how BK currents affect neuronal firing. J Comput Neurosci 30:211-223. CrossRef Medline

Manor Y, Nadim F, Abbott LF, Marder E (1997) Temporal dynamics of graded synaptic transmission in the lobster stomatogastric ganglion. J Neurosci 17:5610-5621. Medline

Marder E (2012) Neuromodulation of neuronal circuits: back to the future. Neuron 76:1-11. CrossRef Medline

Marder E, Bucher D (2007) Understanding circuit dynamics using the stomatogastric nervous system of lobsters and crabs. Annu Rev Physiol 69:291-316.CrossRef

Marder E, O'Leary T, Shruti S (2014) Neuromodulation of circuits with variable parameters: single neurons and small circuits reveal principles of state-dependent and robust neuromodulation. Annu Rev Neurosci 37:329-346. CrossRef Medline

McCormick DA (1989) Cholinergic and noradrenergic modulation of thalamocortical processing. Trends Neurosci 12:215-221. Medline

Morgan PT, Perrins R, Lloyd PE, Weiss KR (2000) Intrinsic and extrinsic modulation of a single central pattern generating circuit. $J$ Neurophysiol 84:1186-1193. Medline

Morris C, Lecar H (1981) Voltage oscillations in the barnacle giant muscle fiber. Biophys J 35:193-213. CrossRef Medline 
Nadim F, Bucher D (2014) Neuromodulation of neurons and synapses. Curr Opin Neurobiol 29C:48-56. CrossRef Medline

Nadim F, Brezina V, Destexhe A, Linster C (2008) State dependence of network output: modeling and experiments. J Neurosci 28: 11806-11813. CrossRef Medline

Nicoll RA (1988) The coupling of neurotransmitter receptors to ion channels in the brain. Science 241:545-551. Medline

Plaza SM, Scheffer LK, Chklovskii DB (2014) Toward large-scale connectome reconstructions. Curr Opin Neurobiol 25:201-210. CrossRef Medline

Prinz AA, Bucher D, Marder E (2004) Similar network activity from disparate circuit parameters. Nat Neurosci 7:1345-1352. CrossRef Medline

Rinzel J, Ermentrout B (1998) Analysis of neural excitability and oscillations. In: Methods in neuronal modeling: from ions to networks, 2 Edition (Koch C, Segev I, eds), pp 251-291. Cambridge: MIT.

Rodgers EW, Krenz WD, Baro DJ (2011a) Tonic dopamine induces persistent changes in the transient potassium current through translational regulation. J Neurosci 31:13046-13056. CrossRef Medline

Rodgers EW, Fu JJ, Krenz WD, Baro DJ (2011b) Tonic nanomolar dopamine enables an activity-dependent phase recovery mechanism that persistently alters the maximal conductance of the hyperpolarization-activated current in a rhythmically active neuron. J Neurosci 31:16387-16397.

Shinkai Y, Yamamoto Y, Fujiwara M, Tabata T, Murayama T, Hirotsu T, Ikeda DD, Tsunozaki M, lino Y, Bargmann CI, Katsura I, Ishihara $\mathrm{T}$ (2011) Behavioral choice between conflicting alternatives is regulated by a receptor guanylyl cyclase, GCY-28, and a receptor tyrosine kinase, SCD-2, in AIA interneurons of Caenorhabditis elegans. J Neurosci 31:3007-3015. CrossRef Medline

Skinner FK, Turrigiano GG, Marder E (1993) Frequency and burst duration in oscillating neurons and two-cell networks. Biol Cybern 69:375-383. Medline
Skinner FK, Kopell N, Marder E (1994) Mechanisms for oscillation and frequency control in reciprocally inhibitory model neural networks. J Comput Neurosci 1:69-87. Medline

Soto-Trevino C, Rabbah P, Marder E, Nadim F (2005) Computational model of electrically coupled, intrinsically distinct pacemaker neurons. J Neurophysiol 94:590-604. CrossRef Medline

Stein W (2009) Modulation of stomatogastric rhythms. J Comp Physiol a Neuroethol Sens Neural Behav Physiol 195:989-1009. CrossRef Medline

Swensen AM, Marder E (2001) Modulators with convergent cellular actions elicit distinct circuit outputs. J Neurosci 21:4050-4058. Medline

Thirumalai V, Prinz AA, Johnson CD, Marder E (2006) Red pigment concentrating hormone strongly enhances the strength of the feedback to the pyloric rhythm oscillator but has little effect on pyloric rhythm period. J Neurophysiol 95:1762-1770. CrossRef Medline

Tsumoto K, Yoshinaga T, lida H, Kawakami H, Aihara K (2006) Bifurcations in a mathematical model for circadian oscillations of clock genes. J Theor Biol 239:101-122. CrossRef Medline

Turrigiano G, LeMasson G, Marder E (1995) Selective regulation of current densities underlies spontaneous changes in the activity of cultured neurons. J Neurosci 15:3640-3652. Medline

Wang XJ, Rinzel J (1992) Alternating and synchronous rhythms in reciprocally inhibitory model neurons. Neural Comp 4:8497.CrossRef

Wu JS, Vilim FS, Hatcher NG, Due MR, Sweedler JV, Weiss KR, Jing $J$ (2010) Composite modulatory feedforward loop contributes to the establishment of a network state. J Neurophysiol 103:21742184. CrossRef Medline

Zhang Y, Bose A, Nadim F (2009) The influence of the a-current on the dynamics of an oscillator-follower inhibitory network. SIAM J Appl Dyn Syst 8:1564-1590. CrossRef Medline

Zhang H, Rodgers EW, Krenz WD, Clark MC, Baro DJ (2010) Cell specific dopamine modulation of the transient potassium current in the pyloric network by the canonical D1 receptor signal transduction cascade. J Neurophysiol 104:873-884. CrossRef Medline 